\title{
Development and Implementation of Wireless Multigas Concentration Cloud System
}

\author{
Sheng-Chung Tien, ${ }^{1}$ Robert Lin, ${ }^{2}$ Tsung-Yu Lee, ${ }^{1}$ Ren-Guey Lee, ${ }^{1}$ and Shu-Ying Huang ${ }^{1}$ \\ ${ }^{1}$ Department of Graduate Institute of Computer and Communication Engineering, National Taipei University of Technology, \\ Taipei 106, Taiwan \\ ${ }^{2}$ Department of Electrical Engineering, Lunghwa University of Science and Technology, Taoyuan 333, Taiwan
}

Correspondence should be addressed to Ren-Guey Lee; evans@ntut.edu.tw

Received 8 December 2013; Accepted 1 January 2014; Published 4 March 2014

Academic Editors: L. Reggiani and A. Song

Copyright (C) 2014 Sheng-Chung Tien et al. This is an open access article distributed under the Creative Commons Attribution License, which permits unrestricted use, distribution, and reproduction in any medium, provided the original work is properly cited.

According to the report "Global Health Risks of 2004" issued by WHO (World Health Organization) in Geneva 2009 (WHO, 2012), the mortality rate of the diseases and cause of death which results from the smoke produced by burning solid fuel is ranked tenth, occupying 3.3\% of the global amounts, and the DALYs (Disability Adjusted Life Years) is ranked the ninth, occupying $2.7 \%$ of the world. Once the pollution occurred in an insufficient ventilation location, the womankind and children will be the most directly affected; therefore, we hoped to focus the measurement environment on indoor surroundings in this paper. This paper proposes a pointing at several kinds of indoor pollutants and integrating multigas sensors, which includes carbon monoxide, carbon dioxide total volatile organic compounds, and so forth. Our paper combines gas sensors with WSN (Wireless Sensor Networks) nodes; we provide users with optional monitoring subjects for constructing and also adapt ZigBee and Wi-Fi modules to get united with uploading real-time sensor messages. Moreover, for the reason of decreasing installation cost of sensors and setting up easily, this paper builds a cloud data-viewing platform additionally, enabling users to observe air quality through the cloud server everywhere.

\section{Introduction}

Though the technology progress is in thriving and robust, human's overexploitation results in natural disasters to happen frequently; it is an extreme threaten to our living environment, so that awareness of environmental protection is rising recently, and the environmental protection issues get much more attention; one of them is air pollution. The diseases are caused by indoor air pollution such as acute lower respiratory infections, chronic obstructive pulmonary disease, lung cancer, asthma, otitis media, nasopharyngeal cancer, and cataract and cardiovascular diseases; especially the first three items are the most in common. Acute lower respiratory infections usually occurred in young children; according to statistics, there are at least two millions of children who died for this illness every year. In the Middle East, lots of women suffered from chronic obstructive pulmonary disease because of exposing in smoke for a long time. Except for common respiratory tract diseases, there is another illness called "Sick Building Syndrome," usually taking place in large building with air condition, but the syndrome only appears when employee stays in office, including dizziness, coughing, and dry-feeling; they will ease after leaving [1]. They are caused by exposure of the high $\mathrm{CO}_{2}$ concentration causes for a long time [2]. In 2013, Roelofs published a paper which detects carbon dioxide indoor and descript the type of ventilation [3], many papers also advance to monitor different gases while executing indoor air pollution detection $[4,5]$, therefore, a powerful device with muti gas monitoring function is necessary.

Therefore, this paper hoped to construct detecting system by wireless method and also aimed at monitoring indoor pollutants; they are $\mathrm{CO}, \mathrm{CO}_{2}$, and TVOC (Total Volatile Organic Compounds). This research combines several gas sensors with ZigBee-based WSN (Wireless Sensor Networks) nodes, called Super Node, which transfers sensor data to the ZigBee-Wi-Fi gateway; then it will be computed in the 
cloud server with a cloud viewing platform providing users for instant monitoring.

\section{Related Works}

WSN has been used in various environmental monitoring systems, many papers proposed about air pollution and other applications, such as forest fires monitoring, temperature and humidity monitoring in agriculture, and monitoring comprehensive air pollutions emitted by vehicles in urban.

In 2009, Kwon et al. and others combined NDIR $\mathrm{CO}_{2}$ sensors with WSN, constructed it at subway station for remote air quality monitoring [10], and then used B-530 and $\mathrm{H}-550$ in test to compare the results with experiment data. The MCU on the sensor node is Atmega 128L; it combines the sensors, SHT75, and NDIR- $\mathrm{CO}_{2}$, for temperature, humidity, and $\mathrm{CO}_{2}$ concentration detecting; the data will be sent to gateway through CC2420 ZigBee transferring interface. The gateway used WLAN method to transfer data to cloud server, but it still needed to control by the subway administrator for the ventilation equipment.

In 2009, Zhengzhong et al. and others realized a WSNbased living environment monitoring system [11]; the monitoring items included temperature, humidity, luminosity, $\mathrm{CO}$ and $\mathrm{H}_{2}$; this system adapted CPLD (Complex Programmable Logic Device) at transferring part for data transforming, sent data to receiver through RF (Radio Frequency) and then uploaded it to cloud server by UART interface; consequently, the user may catch sensor data by means of PC or PDA.

In 2010, there was a report about "Development of MicroScale Air Quality Monitoring System with WSNs," it issued an automatic data-collecting microscale air quality monitoring system [12] based on WSN, integrated global mobile communication system with Internet for detecting vehicle produced pollutants $\mathrm{CO}$ and nitrogen dioxide.

In 2010, Khedo et al. has provided real-time information about the level of air pollution in these regions and provide alerts in cases of drastic change in quality of air [13].

In 2011, Jeličić et al. built a wireless intelligent gas monitoring system [14], MOX (Metal Oxide Semiconductor) sensor was used in major and collocated with PIR (Pyroelectric Infra Red) sensor as Figure 1 shows, system added JN5148 as WSN sensor node and hoped to decrease the energycost through the Hibernate mode of JN5148; meanwhile, this system completed examination of sensors' power consumption with wireless network working time simulation by using CONTAM.

2011, Professor of Kun Shan University, Ching-Biu Tzeng, and his laboratory had built a ZigBee-based Wireless Sensor Network indoor air quality monitoring system [15]; its architecture is depicted as Figure 2, they detected $\mathrm{CO}_{2}$ by means of DS-IAQ2000 module, used SHT10 for temperature and humidity measurement, and the ZigBee Wireless Sensor Network was constructed through CC2430. In order to understand efficiency and other changes of DS-IAQ2000, the Air Boxx made by $\mathrm{KD}$ was added into experiment.

In the same year, Lu proposed a development of WSNbased urban air quality monitoring system [16]; his research integrated meteorological module, global mobile communication system, and Internet with WSN and aimed at CO vehicle produced detecting.

In 2013, Devarakonda et al. were very creative in the air pollution's collecting of vehicles in urban areas, through the GPS function in smart phones, data of air pollution is able to be transmitted to cloud server for analysis. They present a vehicular-based mobile approach for measuring fine-grained air quality in real time [17].

In the same year, they designed a wireless sensor module that had $\mathrm{CO}_{2}$ concentration, $\mathrm{O}_{2}$ concentration, and the density of fine particle dust in a house. They proposed a monitoring air quality system for the people to change the environment [18]. We know that many prototypes for home monitoring systems have been proposed [19-26].

In particular, the affected degree of indoor pollution is bigger than outdoor pollution to human according to the report of WHO research, shown as Table 1.

In this paper, we will focus on indoor pollutants detecting, and especially the smoke from burning solid fuel is the worst. Among the monitoring system mentioned above, most of them were aimed at detecting temperature, humidity, and $\mathrm{CO}_{2}$, but in our research, we not only detect these indexes but also monitor $\mathrm{CO}$ and TVOC, completing the detecting environment indexes. Our system adapts typical WSN structure to construct air quality monitoring system, depicted as Figure 3; the frontal Super Node and ZigBee-WiFi gateway will upload sensor data to cloud server through wireless AP and also provide administrator with monitoring and controlling display for observing.

\section{System Introduction}

The system structure is depicted in Figure 4; it is divided into 3 main parts:

(A) multigas concentration sensor node (Super Node);

(B) data-collecting gateway (ZigBee-Wi-Fi gateway);

(C) data-transferring format;

(D) cloud viewing platform (cloud server).

The gas sensor data will be transferred to ZigBee-Wi-Fi gateway by matched IEEE802.15.4 ZigBee module and then uploaded onto cloud server through Wi-Fi module on the ZigBee-Wi-Fi gateway for saving and displaying data.

User may observe the real-time air condition by the cloud viewing platform which is built by the cloud-remote instant monitoring system and also able to inquiry past trend of air condition, even to be reference while making decisions for air quality improvement.

This research points at the different output interface of five gas sensors to retrieve and adjust the signal; mean while, the system provides user with connecting, measuring the chosen gas sensor through the Sensor-state chosen and using each kind of interfaces of MCU, which is on Super Node, to capture and digitalize signals, then through the ZigBee wireless module on Super Node to transfer it to ZigBee-Wi-Fi gateway. (See Figure 5.) 
TABLE 1: Global health risk index in environmental pollution [27].

\begin{tabular}{|c|c|c|c|}
\hline Risk & World & Low and middle income & High income \\
\hline \multicolumn{4}{|l|}{ Percentage of deaths } \\
\hline Indoor smoke from solid fuels & 3.3 & 3.9 & 0.0 \\
\hline Unsafe water, sanitation, and hygiene & 3.2 & 3.8 & 0.1 \\
\hline Urban outdoor air pollution & 2.0 & 1.9 & 2.5 \\
\hline Global climate change & 0.2 & 0.3 & 0.0 \\
\hline Lead exposure & 0.2 & 0.3 & 0.0 \\
\hline All five risks & 8.7 & 9.6 & 2.6 \\
\hline \multicolumn{4}{|l|}{ Percentage of DALYs } \\
\hline Indoor smoke from solid fuels & 2.7 & 2.9 & 0.0 \\
\hline Unsafe water, sanitation, and hygiene & 4.2 & 4.6 & 0.3 \\
\hline Urban outdoor air pollution & 0.6 & 0.6 & 0.8 \\
\hline Global climate change & 0.4 & 0.4 & 0.0 \\
\hline Lead exposure & 0.6 & 0.6 & 0.1 \\
\hline All five risks & 8.0 & 8.6 & 1.2 \\
\hline
\end{tabular}

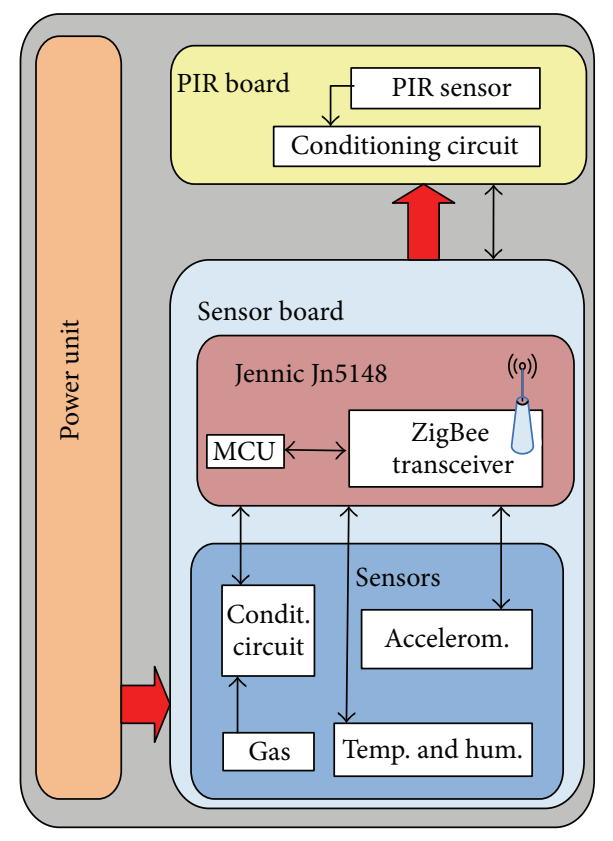

(a) Block architecture

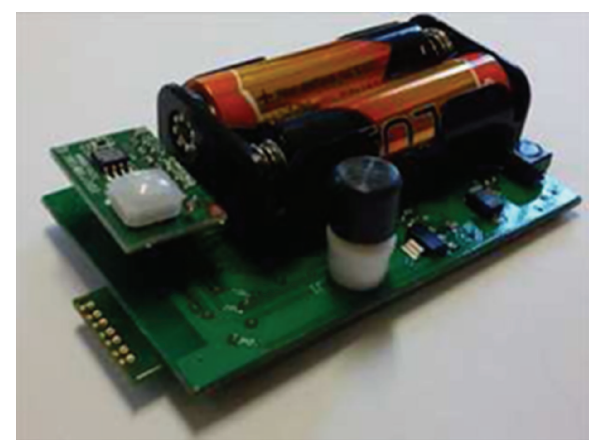

(b) Node with sensors

Figure 1: Node and block diagram [3].

TABLE 2: Correspondence between interfaces and gas sensors.

\begin{tabular}{lcc}
\hline Gas & Sensor & Output interface \\
\hline $\mathrm{CO}_{2}$ & AZ7722 & RS-232 \\
& ZG01 & Digital signal \\
$\mathrm{CO}$ & NAP505 & voltage \\
& MICS5525 & voltage \\
TVOC & TGS2602 & voltage \\
\hline
\end{tabular}

The introduction of sensor part, receiving parts and the cloud viewing platform are as follows.

3.1. Multigas Concentration Node (Super Node). In Super Node part, this paper points at the different output interfaces of five gas sensors to integrate signals with MCU. Table 2 shows correspondence between gas sensors and interfaces; the user may combine the interested gas sensor manually to measure.

The firmware flow chart of multi gas concentration sensor node is illustrated as Figure 6. At first, we will initialize each surrounding part of MCU; the purpose of this motion is automatic detecting which gas sensors have been set up in system.

Next, system will read the "Sensor state" command to discriminate the type of using sensors and then retrieve and analyze data from chosen sensors. At last, system packages data into the transferring package format of ZigBee and sent data to ZigBee-Wi-Fi gateway until receiving the startsending command from it. 


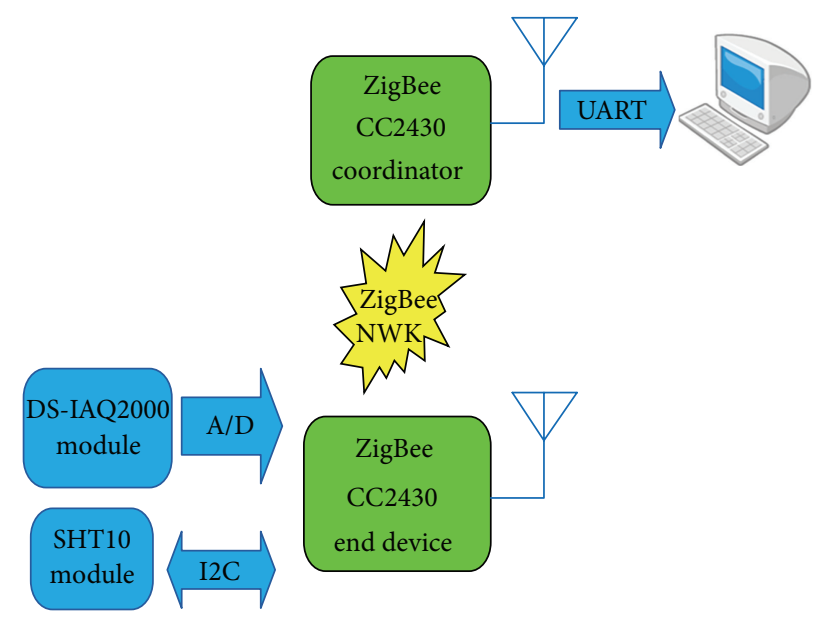

FIGURE 2: Architecture of IAQ monitoring system.

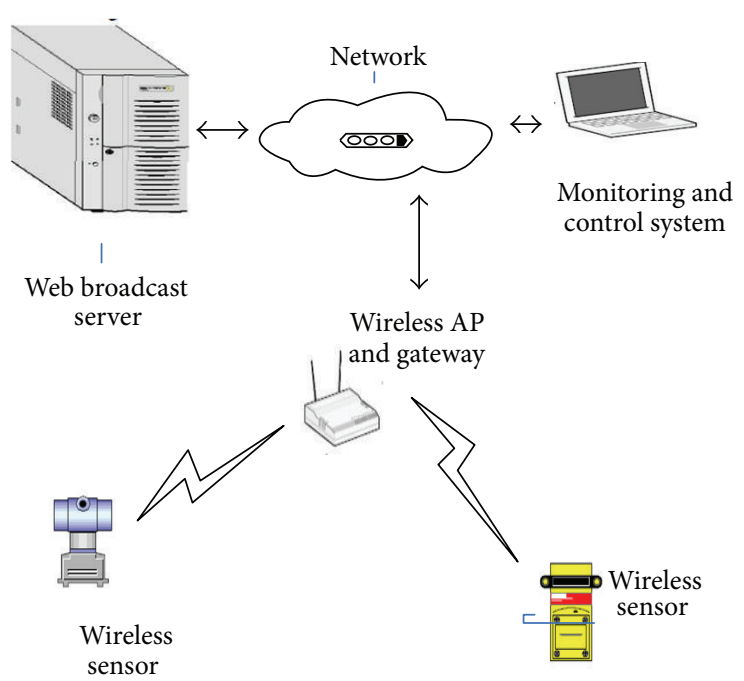

Figure 3: Typical Wireless Sensor Network.

3.2. ZigBee-Wi-Fi Gateway. About the hardware, our system provides $1 * 4$ DIP switches for choosing ZigBee-Wi-Fi gateway communication channel; the range of choosing channels is 11 to 26 ; normally, channel 26 and channel 25 will be chosen for reducing channel interface of IEEE802.11.

On the other hand, the configuration of two $1 * 8$ DIP switches(Node ID state) is provided for setting up usingcondition of sensor node. Under premise of the proper transferring distance, one gateway can be matched with maximum 16 sensor nodes for data transference. Once one of the sensor nodes situation has been set to "ON," the ZigBee-Wi-Fi gateway will timely request sensor data from that sensor node through ZigBee module; after data has been received, system is going to upload data to cloud server for storage by means of wireless Internet connection with Wi-Fi module. Figure 7 is the ZigBee-Wi-Fi structure diagram.

In the software design, ZigBee-Wi-Fi gateway is in charge of timely requesting sensor data from the sensor nodes in using and connecting sensor data to wireless Internet by WiFi module and then uploads it to cloud server.

The ZigBee-Wi-Fi gateway firmware flow chart is shown as Figure 8. At the beginning, the system initializes ZigBee and Wi-Fi modules, continuous reads the channel values and "Node ID State", in order to making users conveniently settle sensors and considering the effects from each environment, this paper has enabled the channel values to be chosen manually to set up a stationary channel in environment arranging; therefore, the sensor data transference will be stable.

The channel value has set to be $\mathrm{CH} 26$ in advance, and the range of channel setting is 11 25, if the influence caused by $\mathrm{Wi}-\mathrm{Fi}$ is not considered, the ideal channel will be 15,20 , and 25 because these three channels have avoided from the Wi-Fi channel. Figure 9 illustrates the comparison diagram of Wi-Fi and ZigBee gateway.

3.3. Data-Transferring Format. In the data-transferring part, our paper uses UZ2400 to be the connecting module between Super Node and ZigBee-Wi-Fi gateway.

UZ2400 is a ZigBee module which corresponding to the module formulated by IEEE802.15.4 and Alliance organization. As shown in Figure 10, UZ2400 is definedin IEEE802.15.4protocol in physical layer and MAC layer, and the other layers above physical layer are formulated by ZigBee Alliance. This ZigBee module supports Star and peer-to-peer topology; it can connect with upmost 255 devices at the same time, in other words, high expanding capability.

In this paper, we use Star topology to construct routing mechanism illustrated as Figure 11. Node only can transfer data with gateway; it is not allowed to connect with other nodes, and the gateway is in charge of collecting each node data.

The format of UZ2400 transferring package is depicted as Table 3. "Frame length" means the length of package; MAC headers is presented as "MAC Header"; "MAC FCS" represents checking sequence of package; "LQI" means the quality of connection; "RSSI" is the strength of signal.

As shown in Table 4, "Area" represents sensor location; "Sensor Type" is the type of this package sender; "0x00" is gateway; "0x01" is sensor node; ID code is presented as "SRC ID"; "DST ID" means receiver's ID code; "SEQ" is the sequence of package, to examine if there is any loss of packages or not.

"TYP" is used for discriminating this package type, such as requesting send-data-back command or send-data-back package; "Check Sum" is the sum of the first 23 "MAC Payload", for correctness of package examination; "RSSI" is the strength of package; and "Payload" is the data byte which can be manually defined; its meaning of data byte in the way where Super Node sent data back to ZigBee-Wi-Fi gateway is depicted in Table 5. All of the "MAC Payload" values can manually set up the representative meaning to make the system have good extensibility; even though we want to add new function in the future.

3.4. Cloud Viewing Platform. Figure 12 represents the software structure diagram of cloud viewing platform in our 


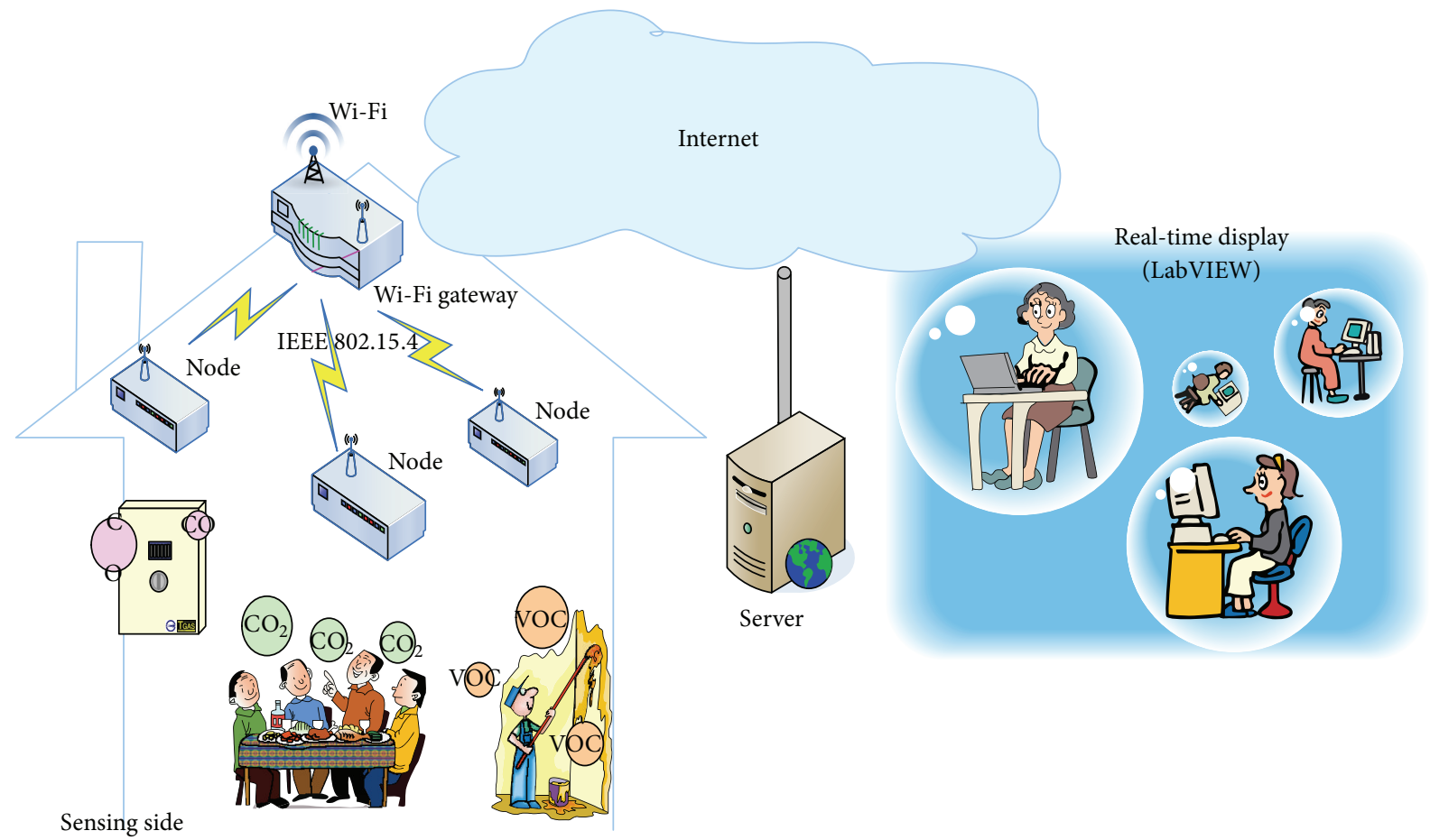

FIgURE 4: System structure diagram.

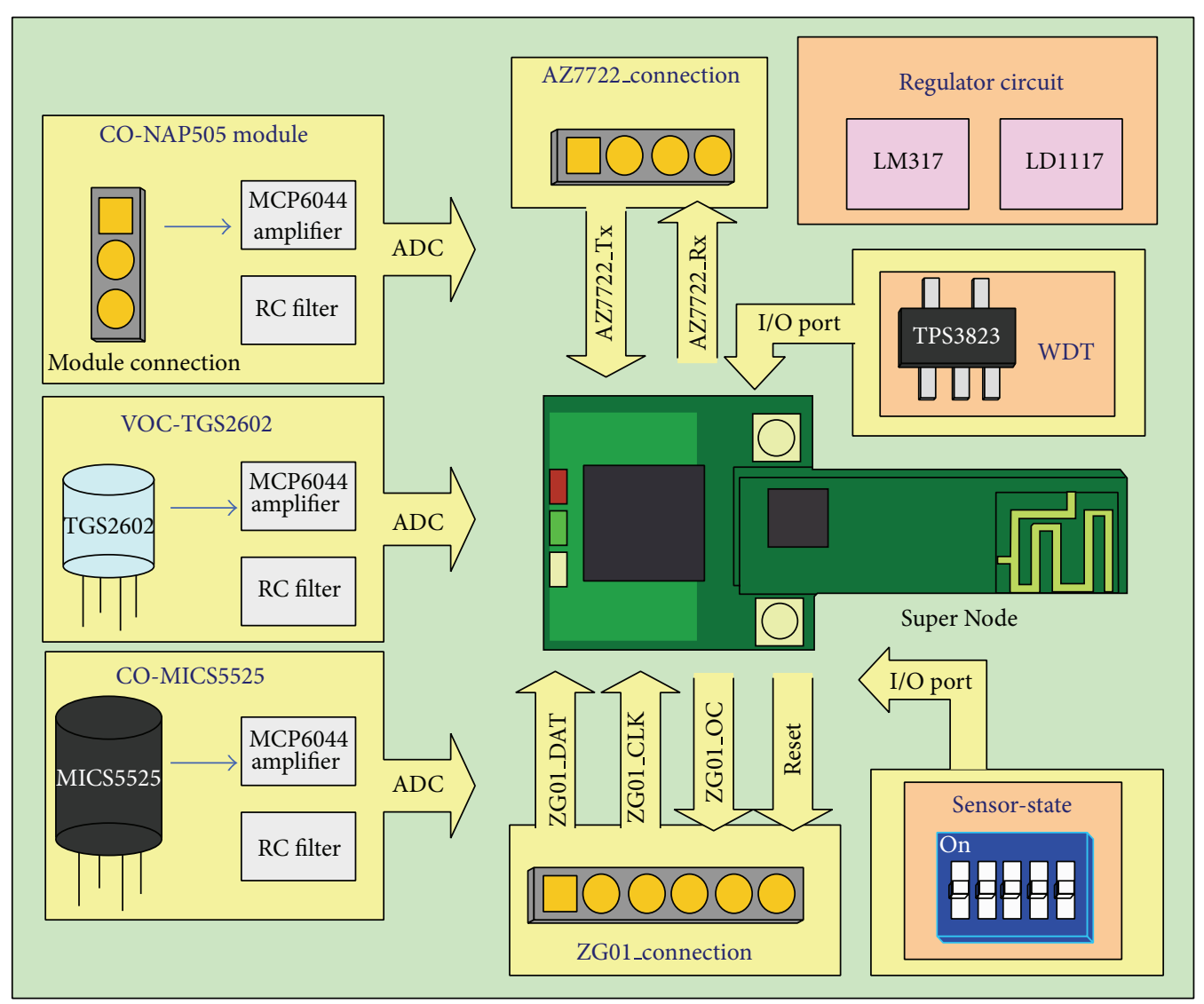

FIgURE 5: The inner structure diagram of multigas concentration sensor node. 
TABLE 3: Data-package format.

\begin{tabular}{|c|c|c|c|c|c|}
\hline 1 byte & M byte & $N$ byte & 2 bytes & 1 byte & 1 byte \\
\hline Frame length & MAC header & MAC payload & MAC FCS & LQI & RSSI \\
\hline
\end{tabular}

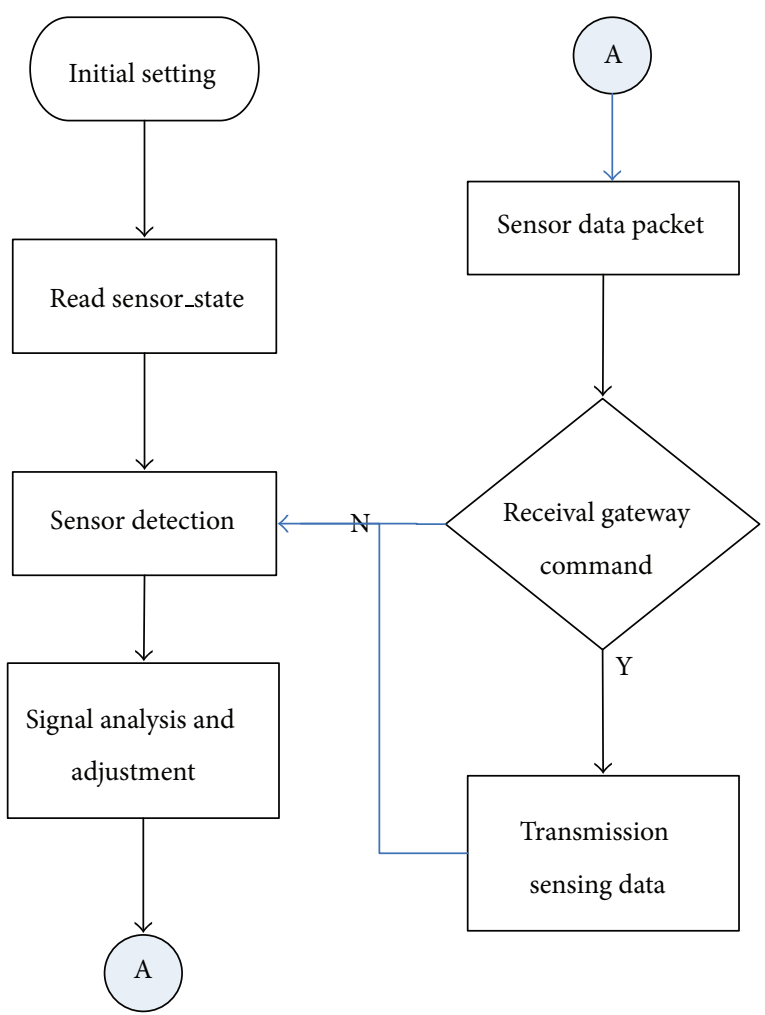

Figure 6: Flow chart of node's firmware.

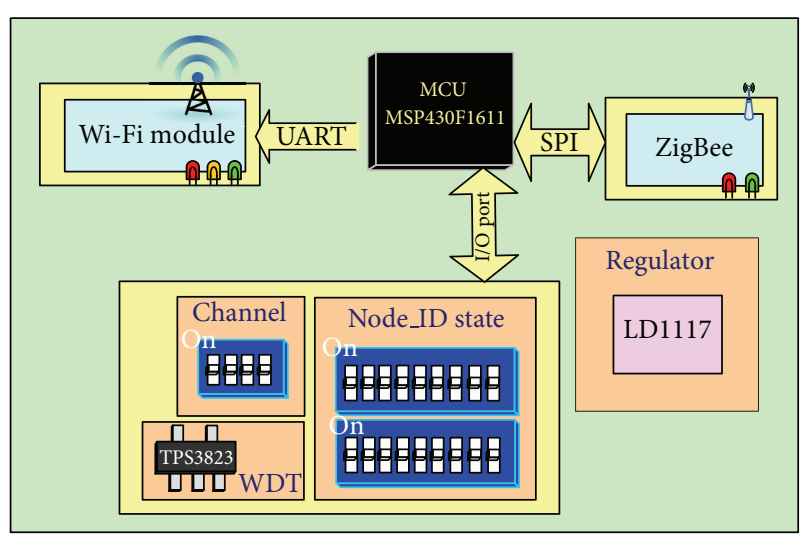

FIGURE 7: Structure diagram of ZigBee-Wi-Fi gateway.

system; it is mainly divided into real-time viewing platform and interface for choosing historical data. It uses different types to classify in real-time cloud viewing platform, such as school, hospital, factory, and store, enabling user to find out location of sensors easier and realize the current concentration. In the interface of choosing historical data, this system provides user to search the data of provisioning
TABLE 4: Package format of MAC Payload.

(a)

\begin{tabular}{|c|c|c|c|c|c|}
\hline 2 bytes & 1 byte & 1 byte & 1 byte & 1 byte & 1 byte \\
\hline Header & Area & Sensor_Type & SRC_ID & DST_IC & SEQ \\
\hline
\end{tabular}

(b)

\begin{tabular}{|c|c|c|c|}
\hline 1 byte & 15 bytes & 1 byte & 1 byte \\
\hline Type & Payload & Check sum & RSSI \\
\hline
\end{tabular}

TABle 5: Definition of data type (Super Node to ZigBee-Wi-Fi gateway).

(a)

\begin{tabular}{lcccc}
\hline 1 byte & 2 bytes & 2 bytes & 2 bytes & 2 bytes \\
Sensor_state & AZ7722_CCO & AZ7722 & AZ7722 & TG01_CO \\
& & Temperature & Humidity & \\
\hline
\end{tabular}

(b)

\begin{tabular}{lcc}
\hline 2 bytes & 2 bytes & 2 bytes \\
NAP505_CO & MICS5525_CO & TGS2602_VOC \\
\hline \multicolumn{2}{l}{ Sensor_state represents the types of chosen sensors. }
\end{tabular}

point and also provides date and time for accessing historical data, being the reference of air quality improvement.

\section{System Validation and Practical Tests}

In order to ensure the efficiency of gas sensors in system, this paper executes the gas sensors examination. It examines the same gas sensor with direct-reading instrument; the results of three kinds of gas sensors are as follows.

4.1. Practical Tests of CO Sensor. Because the CO is a kind of toxic plus its tiny concentration in public, our research chooses to monitor an air sampling bag for observing the sensors changes conveniently. We place the sensor MICS5525, CTSM5module which contains NAP505 sensor and Air box direct-reading instrument in air sampling bag, poured in 100 ppm CO from steel cylinder to test the reacting curve of sensors.

The test results are illustrated in Figure 13, it is an onehour monitoring result after gas pouring and shown as averaged value each minute, all the sensors have reached warm-up-time of sensor. It can be figured out that both of the curves of NAP505 and MICS5525 were rising slowly the same as KD direct-reading instrument five minutes after pouring gas. The reason why NAP505 did not reach 100 ppm is because sensor did not being calibrate previously. The MICS5525 is presented as voltage; it will be tough to fit the transforming formula with exponential form because the 


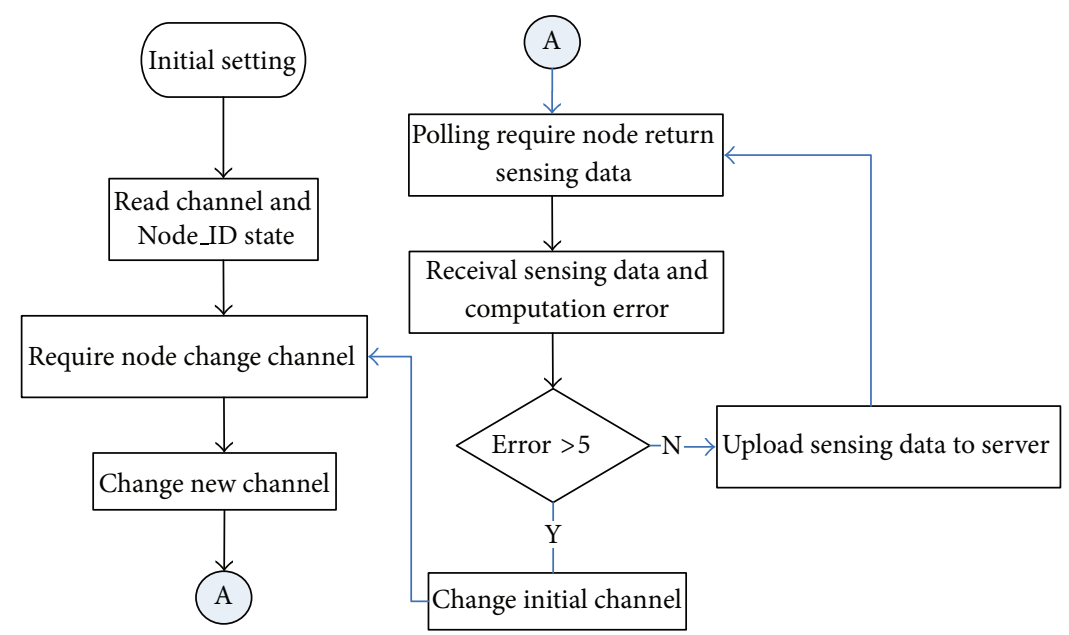

Figure 8: The firmware flow chart of ZigBee-Wi-Fi gateway.

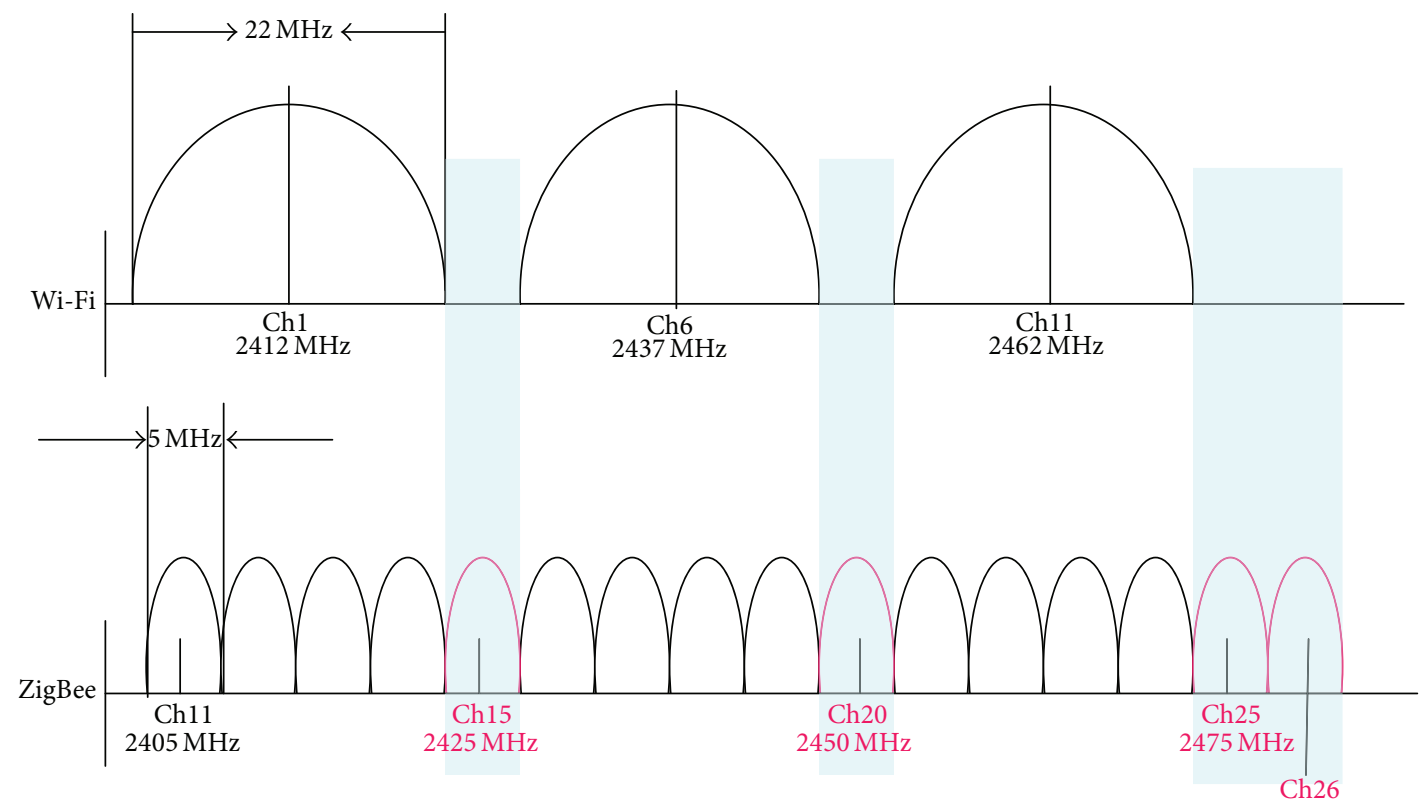

Figure 9: Comparison of Wi-Fi and ZigBee gateway.

voltage of semiconductor sensor will be different after redox reaction.

Furthermore, the difference between red line and green line in Figures 13 and 15 are about being moving-averaged or not; red line's curve is more stable than green one after being moving averaged; it is due to the fact that moving-average can reduce the affect by the pulse.

4.2. Practical Tests of TVOC Sensor. Because TVOC has the feature of condensation and fading, oxygen in test box will decompose VOCs, and VOCs is tend to condense in poorcycling location of test box; therefore, we need to pour in solvent every once in a while to stabilize VOCs concentration. However, when the temperature of the exposure box become rising, the VOCs which previously condensed may evaporate again resulting in concentration's raising. This paper adapts the exposure box made by Fu Jen University, Institute of Public Health, to execute the monitoring of sensors under the consideration of TVOC features.

The environment of TVOC testing exposure box is shown in Figure 14; we tested ToxiRAE Pro produced by RAE Systems Inc. and TGS2602 sensor together in exposure box. Due to the fact that Toluene is ranked first in TVOC organic compounds, this paper pours Toluene for testing the reacting curve of gas sensors in the testing environment of TVOC.

As shown in Figure 15, the testing result diagram of TVOC can found out that there are the same reacting curves of TGS2602 and PID direct-reading instrument. There are different voltages within sensors $\mathrm{A}$ and $\mathrm{B}$; it results from the different amplifying rate on the Super Nodes. Both of 


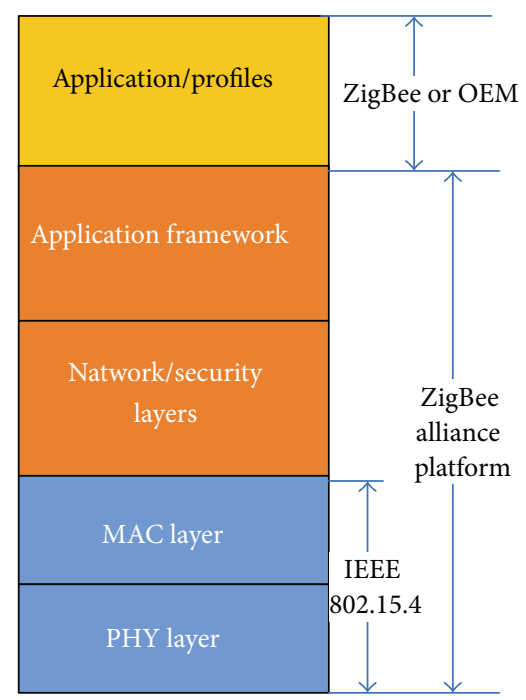

Application

ZigBee platform stack

Silicon

Figure 10: IEEE802.15.4-based ZigBee wireless communication protocol.

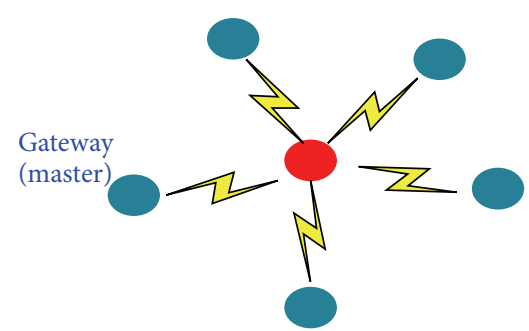

FIgURE 11: Star topology diagram.

TGS2602 and MICS5525 are presented in voltage about the reacting situation.

4.3. Practical Tests of $\mathrm{CO}_{2}$ Sensor. The main reason of indoor $\mathrm{CO}_{2}$ being produced is human's breathing, so that in this research we test in practical circumstance, laboratory of Graduate Institute of Computer and Communication Engineering (volume is about $484.84 \mathrm{~m}^{3}$; 30 people), placing sensor AZ7722, ZG01, and KD direct-reading instrument for comparison, and the height of placement is higher than a person's height to prevent sensor value from human-walking effect. The testing circumstance of $\mathrm{CO}_{2}$ is shown in Figure 16.

Figure 17 is the testing results of $\mathrm{CO}_{2}$, it consists of 24hours continuing monitoring, retrieves one averaged data from half an hour to compare our system with direct-reading instrument,and then executes Linear Regression Analysis with scatter chart. The analyzing result shows that the linear correlation of ZG01 is 0.9958 and AZ7722 is 0.9962; namely, they are extremely close to the comparison between sensor

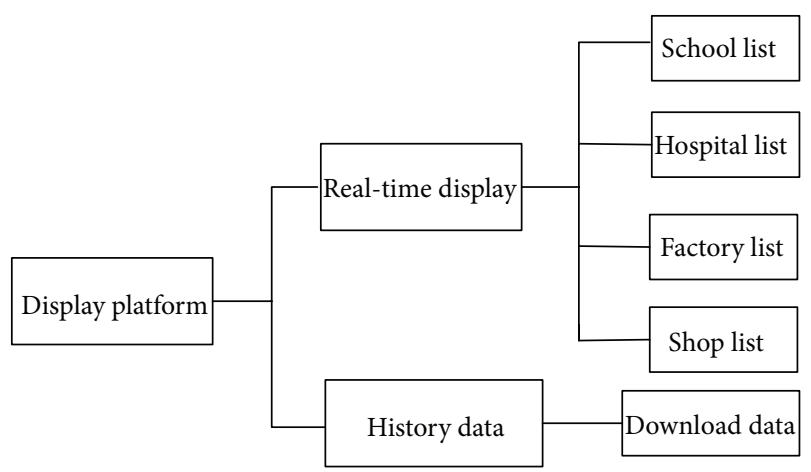

Figure 12: Structure diagram of Cloud Viewing Platform.

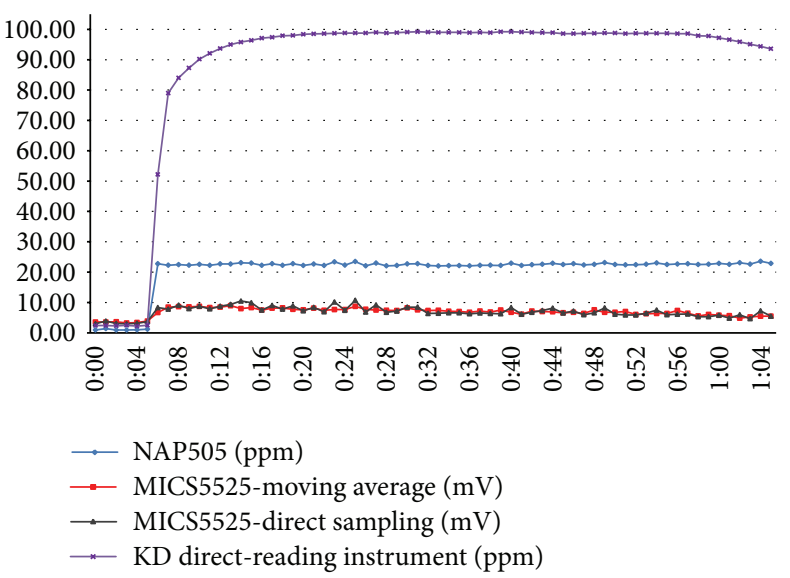

FIGURE 13: Comparison of direct-reading instrument and CO sensor.

concentration and KD direct-reading instrument. Because of people in laboratory has much more amounts and the only ventilated location is the entrance, so that $\mathrm{CO}_{2}$ concentration will be relatively high.

Through the testing results of $\mathrm{CO}$, TVOC, and $\mathrm{CO}_{2}$, it can be figured out that both of AZ7722 and ZG01 the two kinds of $\mathrm{CO}_{2}$ sensors which have better linear reactance, indeed, are reliable to be used in air quality monitoring system.

However, CO and TVOC are presented as trend of reacting curve due to the fact that there is not any stationary transforming equation for concentration; if we want to fit the corresponding equation with exponential form, it will be affected by the redox reactance of semiconductor sensor, each time the resulting voltage will be different because each chemical reactance is not necessarily reverted to be original situation.

Even though the resulting voltage shave some differences between each other, it still can be figured out from the testing result that all of the sensors reacting curves are the same as direct-reading instrument; consequently, if it is going to be used in air quality monitoring system, the alert function for user can be developed by these reacting curves.

4.4. Practical Operation of Cloud Viewing Platform. After constructing sensor nodes has completed, the real-time sensor value can be observed through cloud viewing platform. 


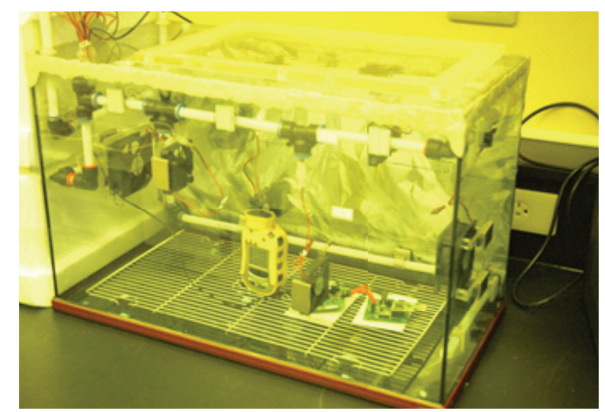

FIGURE 14: Testing circumstance of TVOC.

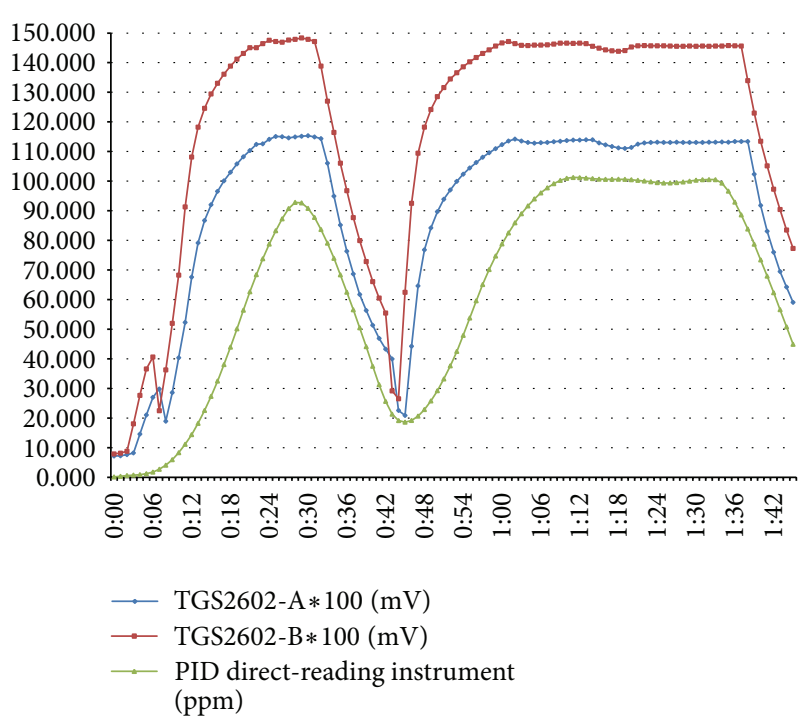

FIGURE 15: Comparison of direct-reading instrument and TVOC sensor.

This paper's classification is according to constructing circumstances; they are school, hospital, factory, and convenient store which provides user with more convenient and faster way to find out sensor nodes by clicking the observing environment of user desire.

If we want to know the school air environment that only needs to choose a school, location of sensors, and ZigBeeWi-Fi gateway, then presses the switch "Observe the latest value" and the updating values of each kinds indexes will be displayed, except for the node which is not built in gas sensor. For now, it will be updated once each five seconds and display the one latest sensor value retrieved from cloud server.

The indoor air quality is not only affecting human-being's health, but also influencing working efficiency if there is not good air quality in office; besides the air management act has been prompted international. However, there is not only one indoor pollutant; since most of gas-monitoring devices can detect one kind of gas only, the cost will become raising if we desire to purchase other kinds of gas sensors.

\section{Conclusions}

Therefore, this paper issues a wireless multi gas concentration monitoring system, which provides three different kinds of

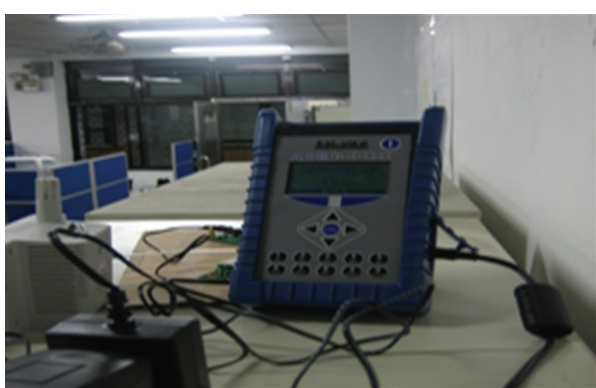

FIGURE 16: Testing circumstance of $\mathrm{CO}_{2}$.

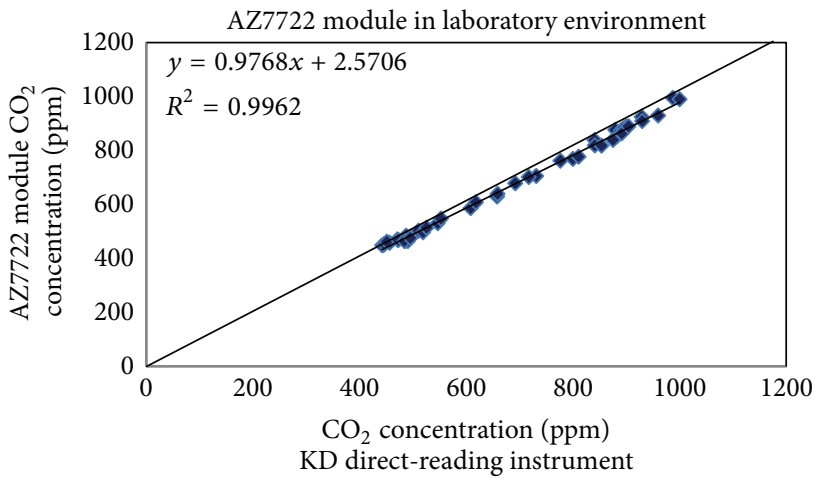

(a) Comparison of AZ7722 module

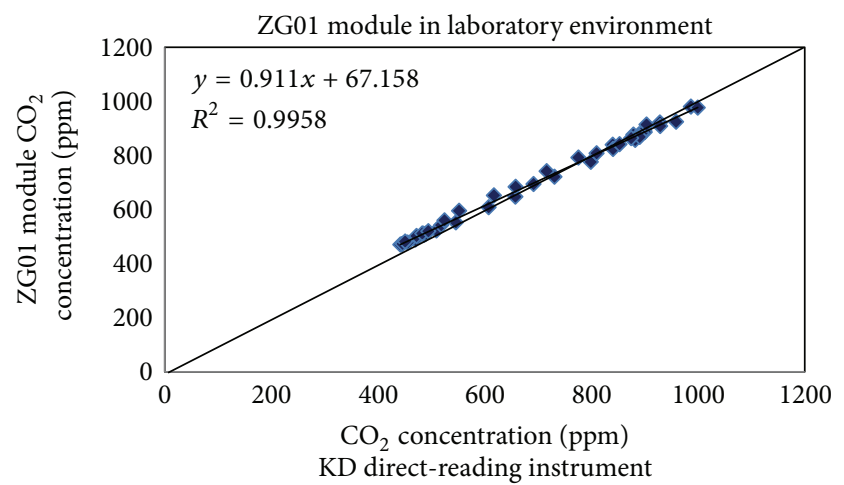

(b) Comparison of ZG01 module

Figure 17: Comparison of $\mathrm{CO}_{2}$ and direct-reading instrument.

gas sensors; users may choose sensor according to their requirement and then utilize the combination of wireless sensor nodes to make it become easier when monitoring multi kinds of sensors at the same time and also add pollutant TVOC which is always being ignored in monitoring, and complete indexes of environment, the air quality of monitoring circumstance will eventually raise.

After summarizing our system in this paper, we conclude these following features.

(1) Monitoring Multigas Simultaneously. Our system provides five kinds of gas sensors connection on the sensor nodes; there are two kinds of gas sensor in $\mathrm{CO}$ and $\mathrm{CO}_{2}$ which are provided for choosing according to the user's requirement, and the sensor nodes will integrate sensor data while using and then transfer data to ZigBee-Wi-Fi gateway, 
so that the changes of each gas kind in the same environment are able to be monitored at the same time.

(2) Wireless Transferring Method. Our system combines WSN to transfer the sensor data of front-end and uploads it through wireless router by $\mathrm{Wi}-\mathrm{Fi}$ connection. The construction of sensors will become easier; we resolve the limit of wired network and reduce routing and cost-raising problem.

(3) Easy to Use and Install. If construct sensor nodes in the distance of radio wave are able to be transferred, except for initializing settings of connection between ZigBee-Wi-Fi gateway and wireless router, the only thing user has to do to execute monitoring is plugging on power line, and then the user can observe the real-time air quality on the cloud viewing platform; it is easy and convenient to use! If user demand to install sensors or replace a new sensor, just follow the marks of sensor nodes on circuit board.

For the future work, we still need to make the sensitivity of system's sensors to be calibrated automatically and build analyzing and alert function in cloud viewing platform and also arrange with professional prediction to provide user with well suggestions and easy-to-understand alerting messages and then improve air quality earlier. We also expect that air quality monitoring can be cross-field integrated, such as combining with vehicular system, prevent user from being in a vehicle environment full of heat-pollutants and monitor the inside-vehicle circumstance to reduce risks by window controlling.

\section{Conflict of Interests}

The authors declare that there is no conflict of interests regarding the publication of this paper.

\section{References}

[1] C. Roelofs, L. S. Azaroff, C. Holcroft, H. Nguyen, and T. Doan, "Results from a community-based occupational health survey of Vietnamese-American nail salon workers," Journal of Immigrant and Minority Health, vol. 10, no. 4, pp. 353-361, 2008.

[2] C. Davidson, Marine Notice: Carbon Dioxide: Health Hazard, Australian Maritime Safety Authority, Canberra, Australia, 2003.

[3] C. Roelofs and T. Do, Exposure Assessment in Nail Salons: An Indoor Air Approach, International Scholarly Research Network Public Health, 2012.

[4] L. J. Goldin, L. Ansher, A. Berlin et al., "Indoor air quality survey of nail salons in Boston," Journal of Immigrant and Minority Health, 2013.

[5] A. Tsigonia, A. Lagoudi, S. Chandrinou, A. Linos, N. Evlogias, and E. C. Alexopoulos, "Indoor air in beauty salons and occupational health exposure of cosmetologists to chemical substances," International Journal of Environmental Research and Public Health, vol. 7, no. 1, pp. 314-324, 2010.

[6] D. D. Chaudhary, S. P. Nayse, and L. M. Waghmare, "Application of wireless sensor networks for greenhouse parameter control in precision agriculture," International Journal of Wireless \& Mobile Networks (IJWMN), vol. 3, no. 1, pp. 140-149, 2011.

[7] Y. Ma, M. Richards, M. Ghanem, Y. Guo, and J. Hassard, "Air pollution monitoring and mining based on sensor Grid in London," Sensors, vol. 8, no. 6, pp. 3601-3623, 2008.
[8] P. Kułakowski, E. Calle, and J. L. Marzo, "Performance study of wireless sensor and actuator networks in forest fire scenarios," International Journal of Communication Systems, 2012.

[9] J. Panchard, Wireless sensor networks for marginal farming in India [Ph.D. thesis], Ecole Polytechnique Fédérale de Lausanne, Lausanne, Switzerland, 2008.

[10] J. Kwon, G. Ahn, G. Kim, J. C. Kim, and H. Kim, "A study on NDIR-based $\mathrm{CO}_{2}$ sensor to apply remote air quality monitoring system," in Proceedings of the ICROS-SICE International Joint Conference 2009 (ICCAS-SICE '09), vol. 33, pp. 1683-1687, August 2009.

[11] W. Zhengzhong, L. Zilin, L. Jun, and H. Xiaowei, "Wireless sensor networks for living environment monitoring," in Proceedings of the WRI World Congress on Software Engineering (WCSE '09), vol. 3, pp. 22-25, May 2009.

[12] T.-Y. Lai, The Development of a Micro-Scale Air Quality Monitoring System Based on WSNs, National Taiwan University, Department of Bio-Industrial Mechatronic Engineering, Taipei, Taiwan, 2009.

[13] K. K. Khedo, R. Perseedoss, and A. Mungur, "A wireless sensor network air pollution monitoring system," International Journal of Wireless \& Mobile Networks (IJWMN), vol. 2, no. 2, pp. 31-45, 2010.

[14] V. Jeličić, M. Magno, G. Paci, D. Brunelli, and L. Benini, "Design, characterization and management of a wireless sensor network for smart gas monitoring," in Proceedings of the 4th IEEE International Workshop on Advances in Sensors and Interfaces (IWASI '11), pp. 115-120, June 2011.

[15] C.-B. Tzeng and T. Wey, "Design and implement a cost effective and ubiquitous air quality monitoring system based on ZigBee wireless sensor network," in Proceedings of the 2nd International Conference on Innovations in Bio-inspired Computing and Applications (IBICA '11), pp. 245-248, December 2011.

[16] H. -C. Lu, The Development of an Air Quality Monitoring System for the Metropolitan Area Based on the Wireless Sensor Network Technology, National Taiwan University, Department of BioIndustrial Mechatronic Engineering, Taipei, Taiwan, 2011.

[17] S. Devarakonda, P. Sevusu, H. Liu, R. Liu, L. Iftode, and B. Nath, "Real-time air quality monitoring through mobile sensing in Metropolitan areas," in Proceedings of the 2nd ACM SIGKDD International Workshop on Urban Computing (UrbComp '13), 2013.

[18] S.-K. Noh, K.-S. Kim, and Y.-K. Ji, "Design of a room monitoring system for wireless sensor networks," International Journal of Distributed Sensor Networks, vol. 2013, Article ID 189840, 7 pages, 2013.

[19] J. Zhang, G. Song, H. Wang, and T. Meng, "Design of a wireless sensor network based monitoring system for home automation," in Proceedings of the International Conference on Future Computer Sciences and Application (ICFCSA '11), pp. 57-60, June 2011.

[20] K. S. Kim, J. G. Lee, S. S. Ahn, and J. Lee, A Study on USNMultiModal Sensor Fusion Technique for Intelligent Air Conversions Digital Home Appliances, MITA, 2010.

[21] K. S. Kim, J. Z. Song, C. Zhang, T. S. Yun, and S. K. Noh, "A study on home indoor environment system," in JCICT \& the 1st Yellow International Journal of Distributed Sensor Networks $7 \mathrm{Sea}$, Proceedings of the International Conference on Ubiquitous Computing (YESICUC '11), 2011. 
[22] M. M. Rahman, M. I. Abdullah, and M. S. Hossain, "Level based path selection technique in large WSN for hierarchical architecture," International Journal of Advanced Science \& Technology, vol. 32, pp. 33-43, 2011.

[23] H. Xianzhe, "Room temperature and humidity monitoring and energy-saving system," in Proceedings of the 6th International Conference on Computer Science and Education (ICCSE '11), pp. 537-540, August 2011.

[24] S. Hwang and D. Yu, "Remote monitoring and controlling system based on ZigBee networks," International Journal of Software Engineering and Its Applications, vol. 6, no. 3, pp. 35-42, 2012.

[25] J. Yap and Y. H. Noh, "A ZigBee-based home control system using OSGi management platform," International Journal of Smart Home, vol. 6, no. 4, pp. 15-28, 2012.

[26] H. Zhang and Y. Liu, "Study and design of adaptive environment control system for mobile base stations," in Proceedings of the International Conference on Control Engineering and Communication Technology (ICCECT '12), pp. 426-429, Liaoning, China, December 2012.

[27] WHO, "Global health risks," 2012, http://www.who.int/healthinfo/global_burden_disease/global_health_risks/en/index.html. 

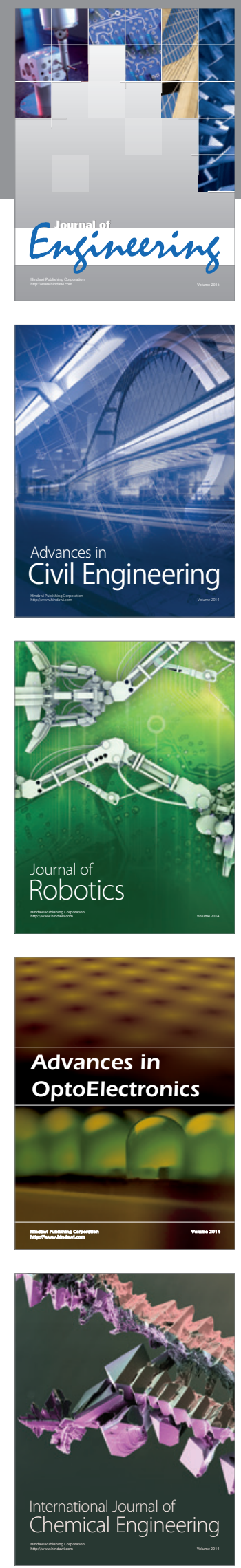

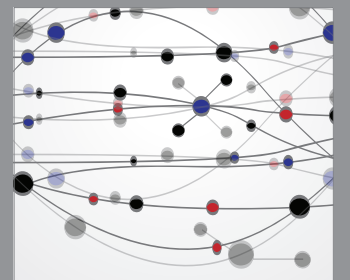

The Scientific World Journal
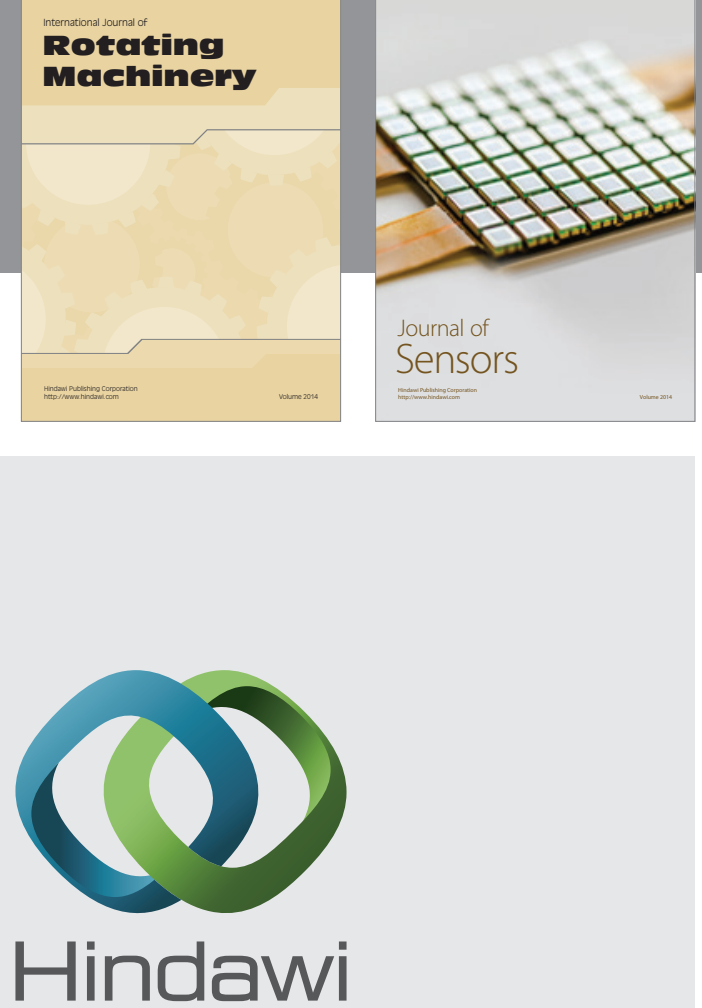

Submit your manuscripts at http://www.hindawi.com
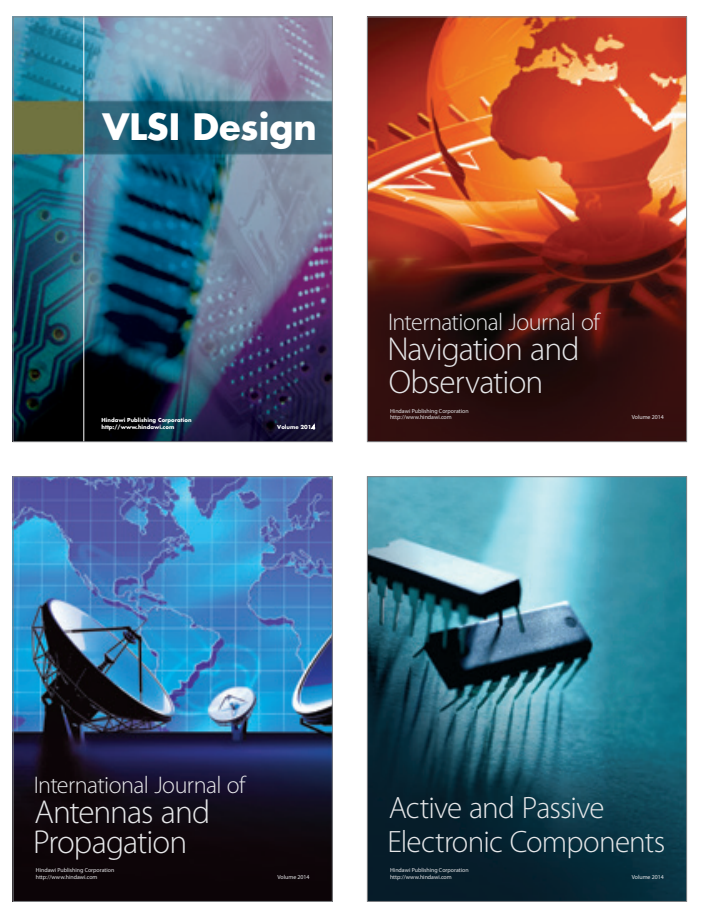
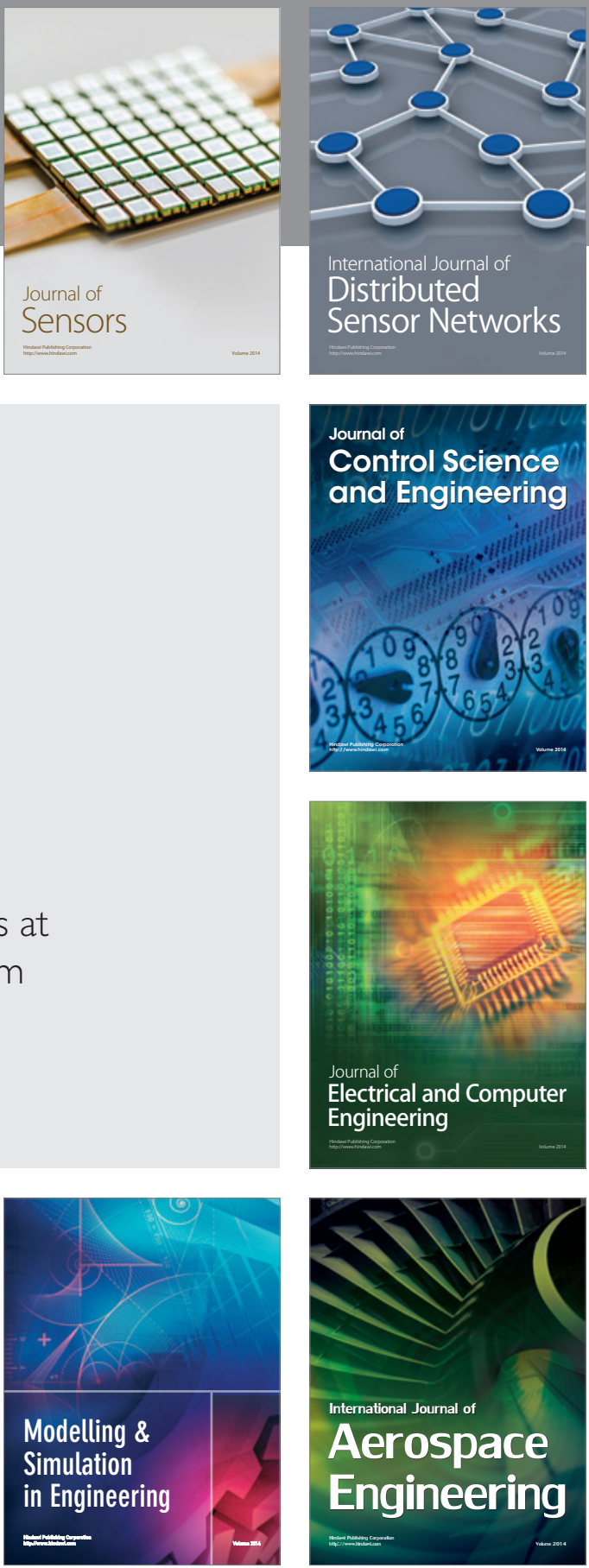

Journal of

Control Science

and Engineering
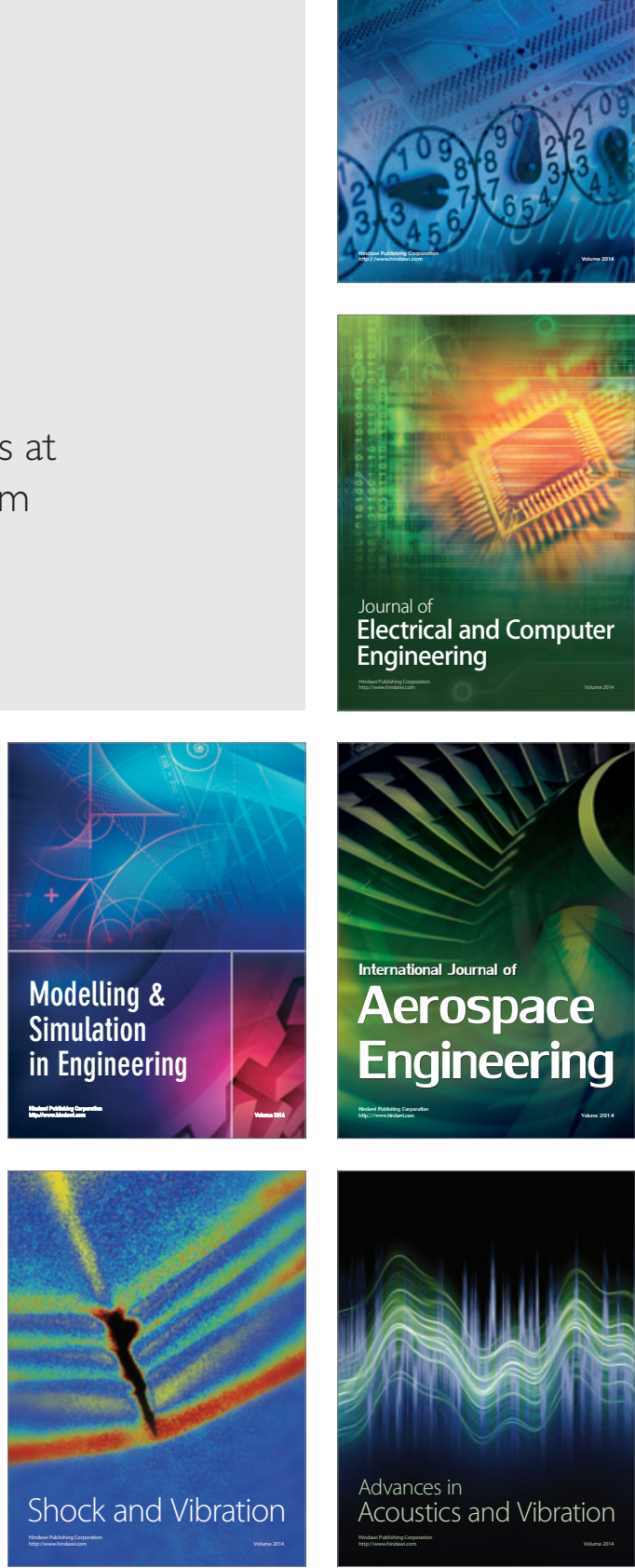